
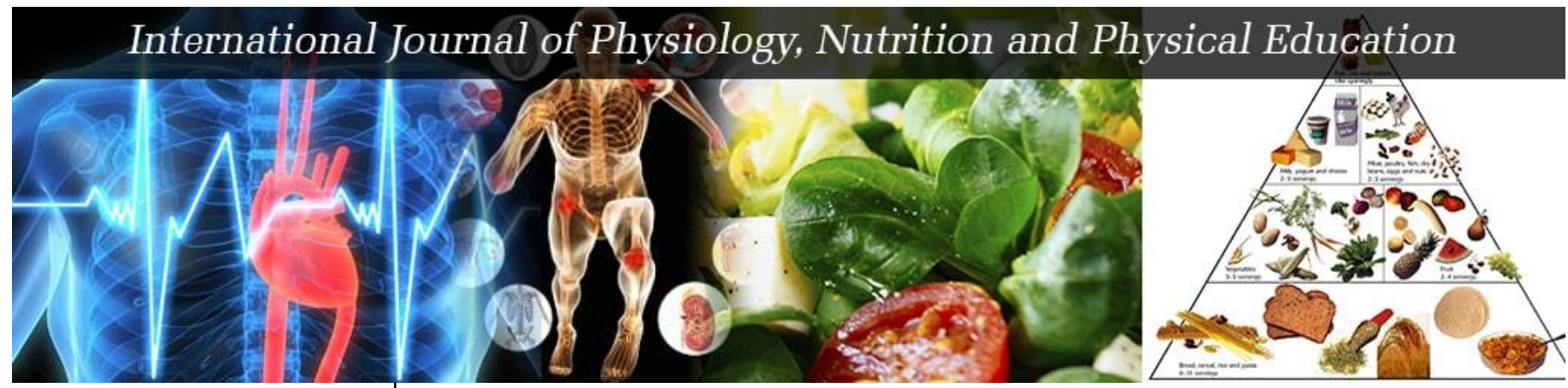

ISSN: 2456-0057

IJPNPE 2020; 5(2): 73-76

(C) 2020 IJPNPE

www.journalofsports.com

Received: 10-05-2020

Accepted: 12-06-2020

Cyanna Joseph D'souza

MPT, Laxmi Memorial College of

Physiotherapy, Mangalore,

Karnataka, India

Sanjay Eapen Samuel

MPT, PhD, Laxmi Memorial

College of Physiotherapy,

Mangalore, Karnataka, India

Celine Julian Fernandes

BPT, Laxmi Memorial College of

Physiotherapy, Mangalore,

Karnataka, India
Corresponding Author: Cyanna Joseph D'souza MPT, Laxmi Memorial College of Physiotherapy, Mangalore,

Karnataka, India

\section{Effect of the intergrated neuromuscular inhibition technique on upper trapezius myofascial trigger points: A case series}

\author{
Cyanna Joseph D'souza, Sanjay Eapen Samuel and Celine Julian \\ Fernandes
}

DOI: https://doi.org/10.22271/journalofsport.2020.v5.i2b.1982

\begin{abstract}
Objective: This study aimed to determine the effectiveness of the Integrated Neuromuscular Inhibition Technique (INIT) for treating Myofascial Trigger Points (MTrPs) in the upper trapezius muscle on outcomes of pain, Range of Motion (ROM) and disability.

Methods: 18 patients mean age $27 \pm$ of 14.78 years were screened for inclusion/exclusion criteria. The INIT was administered three times a week for 4 weeks. The verbal Numerical Pain Rating scale (NPRS), cervical lateral flexion ROM and Neck Disability Index (NDI) were recorded at baseline, 2 and 4 weeks. Results and Conclusion: There were positive changes in all outcome measures for all eighteen participants. An increase in ROM and reduced levels of pain and disability were noted. This case series suggests that a short-term multimodal therapy would be beneficial for individuals with upper trapezius MTrPs. Important preliminary data was collected that will inform more rigorous research in this field of research.
\end{abstract}

Keywords: Case series, integrated neuromuscular inhibition technique, upper trapezius, trigger points, manual therapy

\section{Introduction}

Myofascial Trigger Points (MTrP), an important musculoskeletal derangement is characterized by a specific reference zone of muscle tenderness. It can be defined as a hyperirritable spot along a taut band of skeletal muscle that triggers pain on compression or stretch giving rise to a typical referred pain pattern ${ }^{[1]}$. MTrPs have been associated with limited Range of Motion (ROM) and hyperalgesia and should therefore be addressed as part of a comprehensive physical therapy regimen as they may potentially restrict functional performance ${ }^{[2]}$.

MTrPs are not limited to specific muscle groups, however, the most common sites are levator scapula, upper trapezius, sternocleidomastoid, scalene and quadratus lumborum, all of which actively assist in stabilizing posture ${ }^{[3]}$. An MTrP in the upper trapezius can be a potential contributing factor in conditions like non-specific neck pain where the pathoanatomical source of an individual's pain is difficult to identify ${ }^{[4]}$. The development of an MTrP is hypothesized to result from overstressed or injured muscle fibres that are deprived of glucose, oxygen and other nutrients, that eventually accumulate increased levels of metabolic waste products. The aftermath of the resulting pathogenesis is development of pain, altered tissue status and disability ${ }^{[5,6]}$.

A wide range of physical therapy interventions, both manual and non-manual, are proven to be effective in the deactivation of MTrPs. Ultrasound, acupuncture, botulinum toxin injections are few non-manual approaches, whereas manual techniques involved in the treatment of MTrPs include myofascial release, Muscle Energy Technique (MET), proprioceptive neuromuscular facilitation among many others ${ }^{[7]}$. Chaitow explained how the combination of various manual therapy techniques coined together as the Intergrated Neuromuscular Inhibition Technique (INIT) produced an effective multifaceted approach to MTrP release. This technique involves combining MET, strain-counterstrain (SCS) and ischemic 
compression in a single coordinated manner ${ }^{[8,9]}$. Existing literature including randomized controlled trials have proven the efficacy of MET, SCS and ischemic compression techniques individually in the management of chronic myofascial pain syndromes and neck pain ${ }^{[10,11,12]}$. However, there is a dearth of evidence with regards to the integration of these approaches. Accordingly, additional studies to examine the effect of this integrated regimen is warranted. Therefore, the purpose of this study was to investigate the effectiveness of the INIT in patients with upper trapezius MTrPs on outcomes of pain, ROM and disability.

\section{Materials and methods \\ 2.1 Participants}

In this case series, a total of 18 patients visiting a tertiary hospital in Dakshina Karnataka with a present condition of unilateral upper trapezius MTrPs were recruited. An informed consent was obtained from all participants once they had been screened for suitability and any relevant questions were answered on the procedure and data collection process.

\subsection{Screening process protocol and rationale}

At their first visit, all participants were screened for inclusion/exclusion criteria. Inclusion criteria- patients between ages 18 to 30 years irrespective of gender with active upper trapezius MTrPs or non-specific neck pain (radiating or non-radiating) without any specific systematic disease being detected as the underlying cause of the complaints and less than 3 months duration. Patients responding with a cry, grimace or wince to palpation of the upper trapezius muscle called the 'jump sign'. Exclusion criteria- neck symptoms related to a motor vehicle collision or significant trauma, signs of serious pathology (e.g. malignancy, infection, inflammation, or fracture), signs of cervical spinal cord compromise (e.g. diffuse sensory abnormality, diffuse weakness, hyperreflexia, or the presence of clonus), two or more signs of nerve root involvement (e.g. dermatomal sensation changes, myotomal weakness, or diminished/absent tendon jerk reflexes), history of neck surgery in the past 12 months, history of cervical degenerative joint diseases, endocrine disorders, and autoimmune conditions (e.g. rheumatoid arthritis, fibromyalgia, etc.) or received trigger point injections in the upper trapezius muscle within the past 6 months.

\subsection{Baseline assessment and key outcome measures}

Subjective measures (verbal Numerical Pain Rating Scale (NPRS) and Neck Disability Index (NDI)) and objective outcome measure (cervical lateral flexion ROM) were recorded for each participant at the first baseline assessment and thereafter at intervals of 2 weeks and 4 weeks.

There is evidence to support the construct validity of the verbal NPRS ${ }^{[13]}$. For this, participants indicated the intensity of pain by reporting a number that best represented it, between 0 (no pain) and 10 (maximum pain) ${ }^{[14]}$. The NDI is a 10-item questionnaire which has shown to be a valid and reliable measure of disability in individuals with neck pain (ICC 50.70-0.89) ${ }^{[15]}$. It is scored from $0-50$ points $(0-100 \%)$ in which higher scores correspond to greater levels of disability ${ }^{[15]}$. Cervical lateral flexion ROM was assessed using a universal goniometer which has demonstrated good to excellent inter-rater reliability ${ }^{[16]}$. The shoulder was stabilized to arrest elevation. Subjects were asked to sit upright and laterally flex their head towards the opposite side of involvement and the motion was stopped once the available ROM was completed. Each subject performed three test trials and the average was used for analysis.

\subsection{INIT protocol}

The treatment was provided was a single therapist. Since the primary focus of the INIT approach was to deactivate specific MTrPs, the therapist first identified the MTrPs to be treated within the upper trapezius muscle. The subject was placed in supine to reduce tension in the upper trapezius muscle. The arm was positioned in slight shoulder abduction with the elbow bent and their hand resting on the stomach. Using a pincer grasp, the practitioner moved throughout the fibers of the upper trapezius. Once the MTrP was identified treatment began. The INIT regimen was adapted from a trial conducted by Nagrale et al. ${ }^{[17]}$

The first technique applied was ischemic compression. The therapist again using pincer grasp, placed the thumb and index finger over the active MTrP (Figure 1). Slow, increasing levels of pressure was applied until the tissue resistance barrier was identified. Pressure was maintained until a release of the tissue barrier was felt. At that time, pressure was again applied until a new barrier was felt. This process was repeated either until tension/tenderness was unable to be identified or 90 seconds had elapsed. Similarly, all identified MTrPs were treated.

Ischemic compression was followed by the application of SCS. Moderate digital pressure was applied to the identified MTrP as the subject rated the level of pain using the verbal NPRS. Pressure was increased if pain not identified. If pain was reproduced, the pressure was maintained over the active MTrP as the position of ease was identified. The position of ease was often produced through positioning the muscle in a shortened/relaxed position. Ease was defined as the point where a reduction in pain of at least $70 \%$ was produced. An example of this was supine lying with the head side bent towards the involved side while the practitioner positioned the ipsilateral arm in flexion, abduction and external rotation to reduce the reported MTrP pain (Figure 2). Once the position of ease was identified, it was held for 20-30 seconds and repeated for three to five repetitions.

Lastly, the subject received MET directed towards the involved upper trapezius as per Mohanty's approach ${ }^{[18]}$. With the patient in supine, the therapist placed one hand superior to the affected shoulder and the other hand at the mastoid region (Figure 3). The subject's head was laterally flexed to the opposite side. Once the barrier was reached the subject was asked to lift the shoulder towards the ear while maintaining an isometric contraction with $25-30 \%$ of muscular effort. The contraction was held for about 8-10 seconds. On reaching a new barrier the same method was repeated 6-10 times ${ }^{[18]}$.

All participants were treated three times per week for 4 consecutive weeks and were instructed to maintain their normal activities while avoiding any undue stress to the neck.

\subsection{Data analysis}

Statistical package SPSS (IBM SPSS Statistics for Windows, ver. 21.0. Armonk, NY: IBM Corp.) was used to analyze the data. Descriptive statistics were used to explore the data and included the mean and Standard Deviation (SD) at baseline, 2 weeks and 4 weeks.

\section{Results and Discussion}

Eighteen participants with active upper trapezius MTrPs or non-specific neck pain were recruited (11 females and 7 males); mean \pm SD age and BMI $27 \pm 14.78$ years and $22.96 \pm 5.12 \mathrm{~kg} / \mathrm{m}^{2}$ respectively. At baseline there were a total 
of 22 active MTrPs between 18 participants, on discharge no active MTrPs were found in the upper trapezius.

There was a significant improvement in the outcome measures from baseline to 2 and 4 weeks (Table 1 and 2). A mean difference of approximately 4 points in verbal NPRS between baseline assessment and on discharge was found (Table 2). On discharge the difference in cervical lateral flexion ROM indicated a mean increase of about $5^{\circ}$ (Table 2 ). All participants reported an overall increase in NDI scores. The mean change in NDI at 4 weeks post discharge was around $21 \%$ (Table 2).

The results of this case series indicate significant differences in levels of pain, cervical range of motion and disability associated with neck pain supporting the use of INIT for treating individuals with upper trapezius MTrPs. These findings are consistent with previously published trials on the effect of this approach on MTrPs. Nagrale et al. advocated that INIT was more favorable than METs delivered in isolation to relieve pain, stiffness and ameliorate disability in patients with non-specific neck pain ${ }^{[17]}$. Similar findings were reported by Sabby et al. who concluded that the combination of MET and SCS resulted in improved outcomes of pain and ROM in patients with upper trapezius MTrPs. Rubin et al. reported improved levels of pain and tenderness following osteopathic manipulative treatment (MET and SCS) as an adjunct to medication ${ }^{[19]}$. The current study did not involve any non-manual therapeutic methods, thus allowing for a greater influence of manual therapy on the outcome measures examined. Various other investigations vouch for use of manual therapy for reduction in MTrPs of the upper trapezius, including MET as a major element ${ }^{[20-22]}$.

The delivery of MET mimics the process of autogenic inhibition, influenced by the Golgi tendon organs, in which an isometric contraction is introduced in the affected muscle inducing a post-isometric relaxation ${ }^{[23]}$. This benefits in restoring the length of the tight trapezius by reflex action at the level of the spinal cord ${ }^{[18]}$. However, the additional incorporation of direct ischemic compression and SCS is what makes the INIT more beneficial as compared to using MET. Studies claim that ischemic compression decreases the sensitivity of painful nodules in the muscle and that local pressure may additionally equalize the length of sarcomeres in the involved MTrP thus reducing pain ${ }^{[1,6]}$. The subsequent tissue relaxation created by attaining a position of MTrP ease (SCS) is known to facilitate unopposed arterial filling allowing for tone reduction in the involved tissue. Reduced local tone further results in improved local circulation and modifies neural reporting. Eventually, these alterations promote the resetting of the neural structures, improving resting length and circulation and thus attenuating pain ${ }^{[24]}$. However, these mechanisms need to be further analyzed and examined.

The limitations of this study include a lack of comparison group. Critical in an experimental design, a control group minimizes the influence of extravenous variables on the outcome. Systematic reviews have reported reliability issues on physical examination and manual palpation of MTrP diagnosis thus, creating a need for high MTrP palpation/diagnostic studies [25, 26]. All data collection and interventions were performed by the same individual, posing a risk for researcher bias. Due to the short term follow up, the findings cannot be generalized, thus recommending a loner follow up period to future researchers in further determine the lasting effect of the approach. Although the authors found significant reductions in the outcomes, these findings require a higher level of change to be considered clinically meaningful.

\subsection{Tables and Figures}

Table 1: Mean scores and SD of outcome measures

\begin{tabular}{|c|c|c|c|}
\hline Outcome Measure & Baseline & 2 weeks & 4 weeks \\
\hline NPRS & $5.50 \pm 1.71$ & $3.50 \pm 0.97$ & $1.20 \pm 1.03$ \\
\hline Lateral Flexion ROM (in degrees) & $38.90 \pm 1.91$ & $41.40 \pm 0.97$ & $43.90 \pm 0.74$ \\
\hline NDI (percentage) & $30.41 \pm 12.66$ & $21.02 \pm 7.77$ & $9.03 \pm 3.78$ \\
\hline SD: Standard Deviation; NPRS: Numerical Pain Rating Scale; ROM: Range Of Motion; NDI: Neck Disability Index \\
\hline
\end{tabular}

Table 2: Change scores from baseline to 2 and 4 weeks

\begin{tabular}{|c|c|c|}
\hline Outcome Measure & Difference from baseline assessment to 2 weeks & Difference from baseline assessment to 4 weeks \\
\hline NPRS & $2.00 \pm 0.82$ & $4.30 \pm 1.63$ \\
\hline Lateral Flexion ROM (in degrees) & $2.40 \pm 1.43$ & $5.00 \pm 2.16$ \\
\hline NDI (percentage) & $9.39 \pm 6.23$ & $21.38 \pm 12.05$ \\
\hline
\end{tabular}

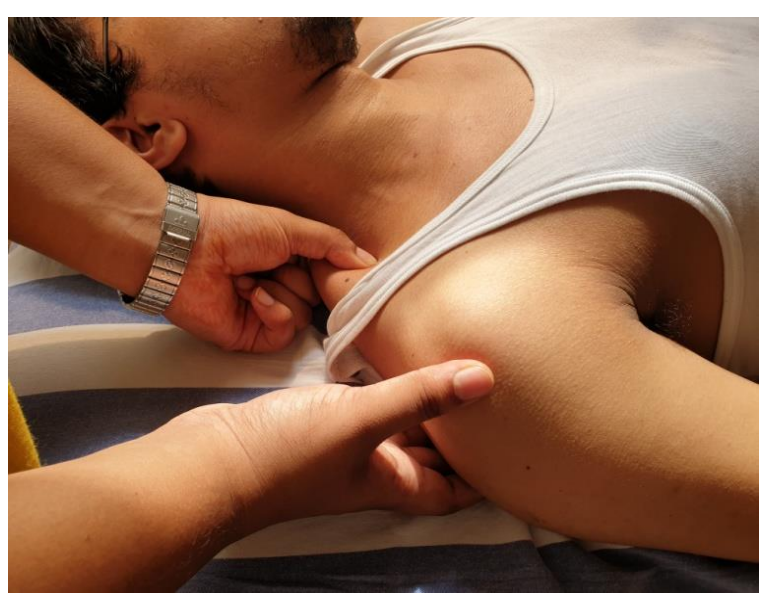

Fig 1: Pincer grasp for Ischemic Compression 


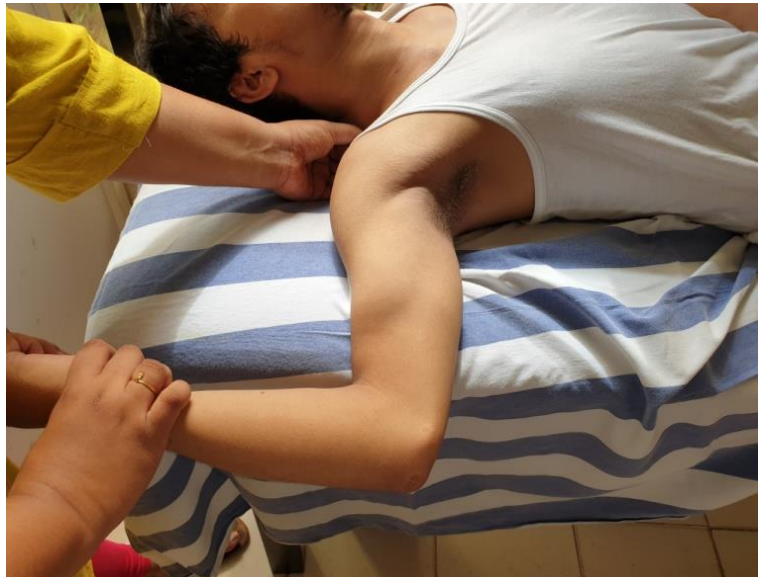

Fig 2: Strain counterstrain technique

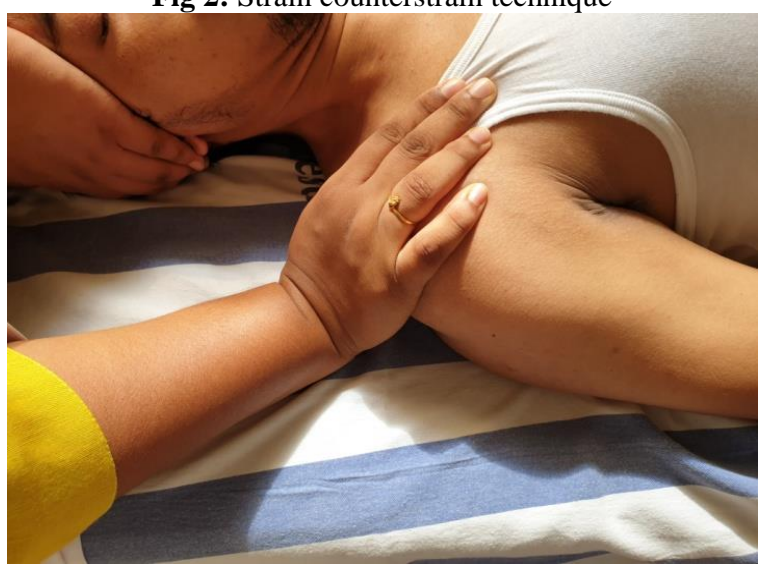

Fig 3: Muscle energy technique

\section{Conclusion}

An integrated approach, such as the INIT, can be useful to reduce pain, improve range of motion and functional ability in patients with upper trapezius MTrPs. These findings support the use of manual therapy in rehabilitation, thus contributing to its growing evidence. The main findings should be accepted by caution given the reliability issues in physical examination and diagnosis of MTrPs. Apart from the identified limitations, this case series will inform more rigorous research in this field of study.

\section{References}

1. Simons DG, Travel J, Simons LS. Myofascial Pain and Dysfunction: The Trigger Point Manual. 2nd ed, Upper Half of Body. Williams and Wilkins, Baltimore, 1999, 1.

2. Harden RN, Bruehl SP, Gass S, Niemiec C, Barbick B. Signs and symptoms of the myofascial pain syndrome: A national survey of pain management providers. Clin $\mathbf{J}$ Pain. 2000; 16(1):64-72.

3. Lavelle ED, Lavelle W, Smith HS. Myofascial trigger points. Anesthesiol Clin. 2007; 25(4): 841-iii.

4. Borghouts JA, Koes BW, Bouter LM. The clinical course and prognostic factors of non-specific neck pain: a systematic review. Pain. 1998; 77(1):1-13.

5. Mense S, Simons DG, Russell IJ. Muscle pain: understanding its nature, diagnosis and treatment. Lippincott Williams \& Wilkins. Philadelphia, PA, 2000.

6. Travell J, Simons DG. Myofascial pain and dysfunction. The trigger point manual. The lower extremities. 1st ed. Lippincott Williams \& Wilkins, Baltimore, MD, 1999, II.

7. Alvarez D, Rockwell P. Trigger points: diagnosis and management. Am Fam Physician. 2002; 65(4):653-60.

8. Chaitow L. Modern neuromuscular techniques. Churchill
Livingstone, Edinburgh, 1996.

9. Chaitow L. Neuro-muscular technique. Thorsons, Wellingborough, 1982.

10. Gemmel H, Miller P, Nordstrom H. Immediate effect of ischaemic compression and trigger point pressure release on neck pain and upper trapezius trigger point: a randomized controlled trial. Clin Chiropract 2008; 11(1):30-6.

11. Hanten WP, Olson SL, Butts NL, Nowicki AL. Effectiveness of a home program of ischemic pressure followed by sustained stretch for treatment of myofascial trigger points. Phys Ther. 2000; 80(10):997-1003.

12. Dardzinski JA, Ostrov BE, Hamann LS. Myofascial pain unresponsive to standard treatment. Successful use of a strain and counterstrain technique with physical therapy. J Clin Rheumatol. 2000; 6(4):169-74.

13. Briggs M, Closs JS. A descriptive study of the use of visual analogue scales and verbal rating scales for the assessment of postoperative pain in orthopaedic patients. J Pain Symptom Manage. 1999; 18(6):438-46.

14. Marquie L, Duarte LR, Marine C, Lauque D, Sorum PC. How patients and physicians rate patients' pain in a French emergency department using a verbally administered numerical rating scale and a visual analog scale. Acute Pain. 2008; 10(1):31-7.

15. Vernon H, Mior S. The neck disability index - a study of reliability and validity. J Manipulative Physiol Ther. 1991; 14(7): 409-15.

16. Youdas JW, Carey JR, Garrett TR. Reliability of measurements of cervical spine range of motioncomparison of three methods. Phys Ther. 1991; 71(2):98106.

17. Nagrale AV, Glynn P, Joshi A, Ramteke G. The efficacy of an integrated neuromuscular inhibition technique on upper trapezius trigger points in subjects with nonspecific neck pain: a randomized controlled trial. J Man Manip Ther. 2010; 18(1):23-8.

18. Umashankar Mohanty. Clinical symposia in manual therapy. Ed 1. MTFI Healthcare Publications. India. 2017.

19. Rubin BR, Gamber RG, Cortez CA, Wright TJ, Shores J, Davis G. Treatment options in fibromyalgia syndrome. J Am Osteopath Assoc. 1990; 90(1):844-5.

20. Delany JP. Foundational platform of NMT. FSMTA Convention. 2007, 08.

21. Chaitlow L, Delany J. Clinical application of neuromuscular techniques. The upper body. Amsterdam: Elsevier; 2008, 1.

22. Goldenberg D. Fibromyalgia, chronic fatigue syndrome and myofascial pain syndrome. Curr Opin Rheumatol. 1993; 5(2):199-208.

23. D'Ambrogio KJ, Roth GB. Assessment and treatment of musculoskeletal dysfunction. Positional Release Therapy. 4th ed. Mosby Publishers, 1997, 164-6.

24. Jones LH. Strain and counterstrain. Colorado Springs, CO: The American Academy of Osteopathy, 1981.

25. Lucas N, Macaskill P, Irwig I, Moran R, Bogduk N. Reliability of physical examination for diagnosis of myofascial trigger points: a systematic review of literature. Clin J Pain. 2009; 25(1):80-9.

26. Myburgh C, Larsen AH, Hartvigsen J. A systematic critical review of manual palpation for identifying myofascial trigger points: evidence and clinical significance. Archives of Phys Med Rehabil 2008; 89(6):1169-76. 
